\title{
Philosophiques
}

\section{À la recherche de la source des normes déontologiques}

\section{Sarah Stroud}

Volume 28, numéro 1, printemps 2001

La nature des normes

URI : https://id.erudit.org/iderudit/004989ar

DOI : https://doi.org/10.7202/004989ar

Aller au sommaire du numéro

Éditeur(s)

Société de philosophie du Québec

ISSN

0316-2923 (imprimé)

1492-1391 (numérique)

Découvrir la revue

Citer cet article

Stroud, S. (2001). À la recherche de la source des normes déontologiques.

Philosophiques, 28(1), 151-171. https://doi.org/10.7202/004989ar

\section{Résumé de l'article}

La pensée morale ordinaire semble incorporer une adhésion à des contraintes ou des restrictions déontologiques : des interdictions qui restent en vigueur même dans des cas où les actions interdites constituent le seul moyen de prévenir des conséquences encore pires. La source de ces normes déontologiques, cependant, n'est pas évidente. Plusieurs tentatives récentes pour trouver une base aux restrictions déontologiques ou pour expliquer ce qui les génère sont examinées. La plus prometteuse insiste sur la valeur intrinsèque du statut moral protégé que la morale déontologique attribue à chacun de nous. On discutera brièvement les points forts d'une telle approche et les difficultés auxquelles elle fait face.
Ce document est protégé par la loi sur le droit d'auteur. L'utilisation des services d'Érudit (y compris la reproduction) est assujettie à sa politique d'utilisation que vous pouvez consulter en ligne.

https://apropos.erudit.org/fr/usagers/politique-dutilisation/ 


\title{
À la recherche de la source des normes déontologiques
}

\author{
SARAH STROUD \\ Université Mcgill \\ sarah@philo.mcgill.ca
}

RÉSUMÉ. - La pensée morale ordinaire semble incorporer une adhésion à des contraintes ou des restrictions déontologiques : des interdictions qui restent en vigueur même dans des cas où les actions interdites constituent le seul moyen de prévenir des conséquences encore pires. La source de ces normes déontologiques, cependant, n'est pas évidente. Plusieurs tentatives récentes pour trouver une base aux restrictions déontologiques ou pour expliquer ce qui les génère sont examinées. La plus prometteuse insiste sur la valeur intrinsèque du statut moral protégé que la morale déontologique attribue à chacun de nous. On discutera brièvement les points forts d'une telle approche et les difficultés auxquelles elle fait face.

ABSTRACT. - Ordinary moral thought seems to reflect a commitment to deontological constraints or restrictions on conduct: to prohibitions which remain in force even if such actions are the only way to avert still worse consequences. The source of these deontological norms, however, is not clear. Several recent attempts to offer a basis for deontological restrictions or to explain what gives rise to them are examined. The most promising such attempt emphasizes the intrinsic value of the protected moral status which deontological morality ascribes to each of us. The strong points of such an approach and the difficulties facing it are briefly discussed.

Un chapitre fort intéressant de la philosophie morale contemporaine a été ouvert par Samuel Scheffler lorsque celui-ci a clairement défini le contraste entre, d'une part, le conséquentialisme et, d'autre part, toute morale comprenant des restrictions déontologiques sur la conduite ${ }^{1}$. Les écrits de Scheffler ont amorcé de part et d'autre une vive discussion qui est toujours en cours. Le conséquentialisme présente les grands avantages de la simplicité et de la clarté structurelle et conceptuelle, mais sa conception de ce qui est moralement permis et de ce qui est interdit ressemble souvent très peu à nos convictions morales fermes. Le déontologisme profite donc d'un lien beaucoup plus étroit avec notre pensée morale : mais, en revanche, il lui manque la transparence conceptuelle dont bénéficie sa rivale. En particulier, nous ne comprenons pas très bien d'où viennent ces normes déontologiques. Quelle est leur source ? Quelles idées animent la conception morale déontologique et donnent lieu aux prohibitions qui la caractérisent ? Les réponses à ces questions ne sont pas évidentes, mais il faut continuer à les chercher, car en

1. Scheffler, 1982.

PHILOSOPHIQUES 28/1 — Printemps 2001, p. 151-171 
comprenant mal les normes déontologiques, nous comprenons mal notre propre conception morale.

Le conséquentialisme en tant que théorie morale se réduit en fait à une seule norme générale qui gouverne toute conduite. Le conséquentialisme base la classification morale de chaque acte uniquement sur ses conséquences (d'où son nom). Notamment, il nous prescrit d'accomplir en toutes circonstances l'acte qui, parmi toutes les lignes de conduite qui nous sont disponibles, aura les meilleures conséquences. En évaluant les états de choses qui résultent d'une action, le conséquentialisme peut utiliser n'importe quelle méthode impartiale et neutre-quant-à-l'agent. Suivant la première condition, les intérêts de chaque personne impliquée dans la situation doivent peser également dans le calcul du meilleur état de choses global : suivant la seconde, les valeurs comptées et calculées doivent être objectives et ne pas dépendre de la perspective particulière de l'agent. La forme la plus familière du conséquentialisme est l'utilitarisme, qui se sert du total du bien-être collectif comme seul critère d'évaluation des conséquences de différentes actions. Mais le conséquentialisme peut être marié à n'importe quelle axiologie qui répond aux conditions déjà notées. L'idée constante dans chaque forme du conséquentialisme, c'est qu'il faut toujours maximiser la valeur (quelle qu'elle soit) en agissant. Il faut toujours choisir l'action dont le résultat - vu d'une perspective impartiale et neutre-quant-à-l'agent — sera le meilleur à tous les points de vue.

Par contraste, un élément important de la pensée morale ordinaire est l'idée que certaines formes de conduite ou de traitement des personnes sont moralement inacceptables peu importe l'état de choses qui peut en résulter. La plupart d'entre nous ne sommes pas prêts à ne considérer que les conséquences d'une action pour la juger, comme le fait le conséquentialiste. Selon la conception morale intuitive que la majorité d'entre nous partageons, certains types d'actions - tels que le viol, la torture, le meurtre - sont interdits, et peuvent continuer de l'être même quand ils constituent le seul moyen de prévenir des conséquences générales encore pires. Dans la littérature, on appelle de telles prohibitions morales des restrictions ou des contraintes déontologiques ${ }^{2}$. Elles ont une toute autre structure que celle qui caractérise le conséquentialisme. Comme l'explique Charles Fried :

C'est parce qu'il est mal (wrong), et pas seulement mauvais (bad), de mentir ou de tuer que ce sont des choses qu'on ne doit pas faire. Ce ne sont pas seulement des négatifs qui entrent dans un calcul et qui peuvent être compensés par le bien que vous puissiez faire ou par le plus grand mal que vous pourriez éviter. Ce caractère absolu est une expression de la manière dont les normes ou les jugements déontologiques diffèrent de leurs contreparties conséquentialistes. ${ }^{3}$

2. La terminologie suivante est également utilisée : restrictions (ou contraintes) centrées sur l'agent ou relatives-à-l'agent.

3. Fried, 1978, p. 9-10. 
En réfléchissant sur nos jugements moraux, nous y trouverons probablement des éléments ayant cette structure (c'est-à-dire des éléments déontologiques) jouant un rôle important dans notre conception morale intuitive. Un exemple de Scheffler, légèrement modifié, nous aidera à voir la différence entre les conceptions conséquentialistes et déontologiques ${ }^{4}$. Un groupe terroriste est sur le point de lancer un attentat à la bombe qui blessera et tuera plusieurs personnes. Le seul moyen de prévenir cet attentat est de torturer l'enfant d'un des terroristes afin que ce dernier communique aux autorités les renseignements qui leur permettront de désamorcer la bombe. Le conséquentialiste dirait, semble-t-il, que le choix correct dans cette situation est de torturer l'enfant. En effet, le résultat de cet acte sera beaucoup moins mauvais que celui de la bombe, vu d'une perspective impartiale. Un grand nombre de personnes estropiées ou tuées constitue un état de choses bien pire qu'un seul enfant blessé (ou même tué). Dans ce cas, le conséquentialisme conçoit la torture comme nécessaire et en commande l'exécution afin d'éviter le premier scénario.

Si cette conclusion nous répugne, c'est que nous avons l'impression d'être confrontés à une norme puissante qui interdit de telles conduites. Comme l'explique Thomas Nagel, celle-ci ne nous apparaît pas comme une simple inhibition psychologique :

Il semble que nous percevions dans (un tel) cas une raison relative à l'agent extrêmement forte de ne pas blesser une personne innocente. Cela se présente comme la perception d'une vérité normative, pas seulement d'une inhibition psychologique (...). Il nous est presque impossible de considérer cela comme une inhibition simplement utile. ${ }^{5}$

Remarquons que l'élément déontologique de la pensée morale ordinaire se manifeste même si nous nous sentons tiraillés par la nature de ce cas, qui nous apparaît comme un dilemme. Le fait même d'être tiraillé évoque le poids que nous accordons à un principe déontologique considéré comme un facteur important dans la décision. Autrement, il en irait beaucoup plus facilement : une fois les faits pertinents stipulés, la réponse conséquentialiste serait rapidement déduite et apparaîtrait clairement. Nous démontrons donc une adhésion au moins partielle aux contraintes déontologiques, même si nous ne sommes pas sûrs de ce qui constitue la bonne décision dans une telle situation. Il demeure qu'en nous référant à de tels principes déontologiques, nous écartons pour de bon le conséquentialisme, car ce dernier n'a pas de place pour des prohibitions qui restent en vigueur même quand le fait de les respecter entraîne des conséquences moins désirables qu'une violation préventive ${ }^{6}$.

4. Scheffler, 1988, p. 3. Scheffler présente également dans son texte de 1982 un autre type de contraste assez frappant entre le conséquentialisme et la pensée morale ordinaire : celleci accorde à l'agent des "prérogatives » qui sont incompatibles avec les préceptes de celui-là. Nous ne considérons pas ici cet élément de la conception morale intuitive.

5. Nagel, 1986, p. 179 : l'ordre des phrases a été modifié. 


\section{Philosophiques / Printemps 2001}

En contraste avec la transparence de la genèse du principe conséquentialiste, la source des interdictions déontologiques auxquelles nous semblons croire peut paraître obscure. Le conséquentialisme exprime de façon directe l'idéal moral du traitement impartial, faisant appel également aux concepts qui nous sont déjà familiers de par d'autres contextes. Par exemple, nous utilisons déjà l'idée générale de faire ce qui aura le meilleur résultat dans le domaine de la prudence et de la rationalité individuelle. Par contre, les normes déontologiques ne semblent pas faire partie d'un tel cadre de raisonnement. D'où viennent-elles ? Comment expliquer la présence dans la morale des prohibitions de ce genre ? Nous aimerions mieux comprendre ces éléments déontologiques, afin de mieux comprendre nos convictions morales et l'univers moral dans lequel nous vivons. Dans ce texte, donc, nous ferons un bref tour d'horizon de la littérature afin d'examiner et d'évaluer quelques tentatives notables pour expliquer la base ou la source des normes déontologiques. Notre investigation commencera par de brèves discussions de quelques approches qui, bien que fort connues, s'avéreront peu prometteuses.

Dans certains écrits qui ne comptent pas parmi ses plus récents, et notamment dans The View From Nowhere ${ }^{7}$, Thomas Nagel a suggéré que l'on pourrait trouver une explication ou une meilleure compréhension des restrictions déontologiques en supposant que ces restrictions sont particulièrement liées au fait d'accomplir ou de viser le mal. Quand on viole une interdiction déontologique, on incorpore le mal dans ce que l'on fait : voilà la raison pour laquelle on juge que cet acte est « mal ». Mais deux questions s'imposent à propos de cette suggestion. D'abord, il faut demander ce qui constitue exactement faire ou viser le mal. De plus, une fois le concept spécifique choisi (que ce soit faire ou viser ou un autre concept voisin), il faut expliquer plus précisément les conditions nécessaires pour l'application de ce concept à une action. Dans notre recherche d'une explication des normes déontologiques, il faut éviter que les concepts servant présumément à l'explication des prohibitions déontologiques puissent être également utilisés par un adversaire du déontologisme pour motiver une conception conséquentialiste (par exemple). Dans un tel cas, nous saurions que les concepts auxquels nous avons fait appel n'étaient pas suffisamment nets pour soutenir la conception déontologique contre ses rivales. Nous saurions que nous n'avons pas encore trouvé l'explication complète des convictions déontologiques.

Ce danger semble présent dans le cas de la suggestion de Nagel. Car il y a des interprétations conséquentialiste, peut-être plausibles, des concepts dont Nagel fait usage, qui rendraient ces concepts inaptes à expliquer ou à défendre le déontologisme. Considérons à titre d'exemple deux interprétations possibles de ce qui constitue "viser le mal ». (L'intérêt porté ici aux

6. Je voudrais remercier un évaluateur anonyme de Philosophiques pour des commentaires qui m’ont incitée à étoffer ce paragraphe.

7. Nagel, 1986, Chapitre 9. 
intentions et aux buts de l'agent est en fait peu caractéristique du conséquentialisme, mais passons.) Le conséquentialiste pourrait proposer qu'on vise le mal si et seulement si on vise à amener un état de choses moins désirable que le meilleur qu'on aurait pu susciter. Ou encore, le conséquentialiste pourrait préférer réserver le concept de « viser le mal » pour des cas où l'agent vise à diminuer le bien-être global. Le conséquentialiste peut se déclarer d'accord avec Nagel : selon l'une ou l'autre de ces deux interprétations, viser le mal est toujours moralement proscrit. Car le conséquentialisme consiste en une seule instruction aux agents : accomplir l'action qui aboutit au meilleur état de choses possible. La prohibition du fait de "viser le mal », dans n'importe quel sens déjà défini, pourrait donc facilement être considérée comme un corollaire du principe de base du conséquentialisme. Cependant, selon ces nouvelles interprétations de l'idée nagelienne, cette interdiction ne ressemble pas du tout à une contrainte déontologique. Maltraiter quelqu'un intentionnellement ou causer du tort à quelqu'un de façon délibérée ne constituera pas, comme le souhaite Nagel, un cas où l'on " vise le mal ", si on agit ainsi afin d'amener de meilleures conséquences globales que celles qui s'ensuivraient si on n'entreprenait pas cette action.

Même l'exemple introduit par Nagel pour illustrer les contraintes déontologiques ne serait pas un cas où l'on « vise le mal » selon les interprétations conséquentialistes de cette idée que nous avons considérées. Dans l'exemple central de Nagel, le seul moyen d'obtenir les clés d'une voiture dont on a besoin pour transporter des amis blessés à l'hôpital est de tordre le bras du petit-fils de la propriétaire de la voiture. Nagel explique que ce dilemme est facile sous une analyse conséquentialiste : les conséquences de l'action de tordre le bras - y compris, bien sûr, les conséquences souhaitables pour les amis de l'agent concerné — sont bien meilleures, tout compte fait, que celles de ne pas tordre le bras. Tout sentiment qu'il serait mal de tordre le bras à l'enfant dans une telle situation doit donc provenir d'une norme déontologique interdisant une telle conduite bien qu'elle puisse entraîner de meilleures conséquences. Nagel essaie d'expliquer ou de justifier l'idée d'une telle restriction en invoquant une prohibition morale à l'égard de "viser le mal ». Mais selon les interprétations conséquentialistes déjà citées de ce dernier concept, l'acte de tordre le bras à l'enfant ne compterait pas comme un cas où l'on vise le mal. En tordant le bras, on agirait ainsi précisément pour amener les meilleures conséquences possibles dans les circonstances et pour alléger la misère qu'éprouvent les amis de l'agent (augmentant ainsi le bienêtre collectif). Cet acte ne satisferait donc ni l'une ni l'autre des définitions conséquentialistes de viser le mal et ne serait pas interdit par l'idée (que le conséquentialiste peut maintenant accepter) qu'il ne faut jamais viser le mal.

Même en supposant une réponse satisfaisante au problème déjà souligné - c'est-à-dire le besoin d'élaborer suffisamment les concepts utilisés dans une présumée explication pour les distinguer de toute interprétation adverse - une autre question demeurerait. Nagel a-t-il identifié de façon convain- 
cante pourquoi il est mal de traiter quelqu'un d'une manière interdite par une norme déontologique? Avons-nous trouvé une explication intuitivement satisfaisante du mal que nous percevons dans certaines formes de conduite? Nagel essaie d'expliquer son raisonnement comme suit. Quand le mal est notre but, ou le moyen que nous avons choisi pour atteindre un but, nous sommes guidés par le mal. Loin d'être une conséquence regrettable d'un projet souhaitable, le mal est la destination vers laquelle nous avons choisi de nous diriger. Si notre ligne de conduite n'aboutissait pas au mal en question, nous réviserions notre stratégie pour garantir qu'elle entraîne ce mal. Cependant, dit Nagel, " la nature du mal est de devoir nous inspirer de la répulsion ${ }^{8}$. Nous agissons donc "à l'encontre de la fibre normative " quand nous incorporons le mal dans ce que nous faisons. Une telle procédure renverse, intervertit, bouleverse le fonctionnement approprié du raisonnement pratique et de l'action.

Même après cette élaboration, cependant, la question demeure : avonsnous trouvé la raison pour laquelle nous jugeons que certains actes sont mal ? Nagel semble suggérer qu'il est irrationnel, dans un sens générique et non spécifiquement moral, d'agir de certaines manières (c'est-à-dire de rechercher ou viser le mal). Mais il est difficile de comprendre pourquoi les fautes rationnelles d'un agent seraient à la base d'une prohibition morale. Un aspect curieux du raisonnement de Nagel est l'importance exclusive qu'il accorde à l'agent, tandis que nous croyons intuitivement qu'une interdiction morale doit provenir, d'une manière ou d'une autre, du tort qu'un tel acte cause à la victime. Donc, nous ne trouverons probablement pas juste l'explication de Nagel, au regard des convictions morales qui la motivent : elle ne paraît pas analyser correctement la provenance des restrictions auxquelles nous croyons en tant que déontologistes intuitivistes.

Il est possible d'adresser la même critique à la tentative de Stephen Darwall, qu'il développe dans son intéressant article "Agent-Centered Restrictions from the Inside Out ». Dans ce texte, Darwall commence par délinéer une perspective à partir de laquelle la tâche de justifier les contraintes déontologiques paraît insurmontable. Il appelle cette perspective sur l'éthique "l'éthique à partir de l'extérieur » (from the outside in). Puis il décrit une autre perspective, selon laquelle ces restrictions paraissent entièrement naturelles et intelligibles : c'est l'approche " à partir de l'intérieur ». Selon Darwall, le conséquentialisme provient d'un point de vue extérieur à l'agent moral, en ce que sa conception de l'action juste et, à la suite, sa théorie du caractère intègre, sont basées sur une évaluation préalable, neutre-quant-à-l'agent, de la valeur des états de choses. Une telle approche de l'éthique ne facilite pas la justification ou même la compréhension des restrictions déontologiques. En effet, il est difficile de comprendre, à partir d'une perspective qui se préoccupe uniquement de ce qui a lieu, pourquoi il pourrait être moralement interdit de commettre un acte

8. Ibid., p. 163. 
qui, bien que répréhensible, aura pour effet de réduire le nombre total d'actes du même type. Mais les contraintes déontologiques, précisément, interdisent parfois de commettre de tels actes. Il est défendu, selon la perspective déontologique, de torturer une personne, même pour empêcher plusieurs actes de torture de la part d'autres agents. Darwall conclut que nous ne comprendrons jamais ces convictions déontologiques si nous restons enfermés dans une perspective « à partir de l'extérieur » sur l'éthique.

En revanche, selon Darwall, en abordant la morale d'une autre façon, nous verrons facilement d'où viennent les normes déontologiques. Selon l'approche "à partir de l'intérieur » de l'éthique, on se concentre sur le caractère moral de l'agent et on dérive une théorie de l'action juste de la conception qu'en a un individu moral responsable ${ }^{9}$. De ce point de vue, il n'y a rien de mystérieux à ce qu'il importe moralement que ce soit moi qui torture l'enfant dans notre cas original. Nous pourrons finalement comprendre les normes déontologiques, en tant qu'elles sont centrées sur l'agent. Darwall croit même que nous devons voir la morale de cette façon — « à partir de l'intérieur » - pour faire valoir les restrictions déontologiques, que nous n'avons pas d'autre choix. Examinons maintenant comment il entend faire le lien entre la perspective " à partir de l'intérieur » et la conception déontologique de la morale.

Selon Darwall, l'éthique doit prendre pour point de départ le fait de prêter attention au caractère et, en particulier, à l'intégrité morale de l'individu. L'intégrité consiste dans le fait qu' "une personne gouverne sa vie à partir de ses propres jugements moraux $»^{10}$. Quand je délibère et que j'agis conformément à ma conscience, je maintiens mon intégrité morale en tant qu'agent. $\mathrm{Si}$, par contre, j'agis contrairement à mes propres croyances et jugements moraux, je porte atteinte à mon intégrité. L'intégrité en ce sens est cruciale pour Darwall, car pour lui, «la responsabilité fondamentale de la vie morale consiste à maintenir l'intégrité ${ }^{11}$. Voilà donc l'exigence la plus fondamentale à laquelle nous sommes assujettis en tant qu'êtres moraux, le défi de base de la vie éthique. Mon souci central comme agent moral est de préserver mon intégrité morale. À partir de cette insistance sur le caractère, nous pouvons dériver directement le principe suivant, qui articule comment nous devons agir : " les individus doivent se conduire de la manière qui est nécessaire au maintien de leur intégrité morale ${ }^{12}$. Cette idée est le cœur de la conception darwallienne de l'action juste.

À supposer que nous acceptions cette approche de l'éthique, pourquoi penser qu'elle générerait des restrictions centrées sur l'agent ? En fait, on pourrait même se demander pourquoi elle engendrerait, d'une manière ou

9. Darwall, 1986, p. 291.

10. Ibid., p. 305.

11. Ibid., p. 305.

12. Ibid., p. 310. 
d'une autre, une conception déterminée des types de conduite interdits. Cette théorie prescrit aux agents de s'assurer que leurs actions et leurs délibérations s'accordent avec leurs jugements moraux. Elle met l'accent sur « la responsabilité d'un individu (...) de ne pas faire ce qui est mal selon son jugement ${ }^{13}{ }^{\text {» }}$. Donc, au lieu de prescrire ou de proscrire directement certains types d'actions, cette approche exige plutôt qu'une certaine relation tienne toujours entre les actes et les jugements d'un agent. Néanmoins, Darwall croit que cette approche donnera lieu à certaines restrictions déontologiques sur la conduite. Son raisonnement va comme suit. Même les conséquentialistes - tout comme, bien sûr, ceux qui adhèrent à la morale du sens commun — croient que les agents devraient être guidés par des principes qui interdisent certains types d'actions malgré toute conséquence bénéfique possible. Nous croyons tous plus ou moins, dit Darwall, que les principes qui sont aptes à guider les agents dans leurs délibérations morales incluent des restrictions déontologiques. Cependant, dans l'optique conséquentialiste, ce fait n'a pas de rapport direct avec la théorie de l'action juste. Pour le conséquentialiste, les principes qui doivent guider les agents ne déterminent pas ce qui, en fait, est juste, et ce qui est véritablement mal. Plutôt, ces principes sont les meilleurs guides de l'action parce qu'ils sont efficaces sur le plan causal : les agents qui les suivent ont plus de chances de faire le choix d'actions justes, mais qui le sont pour des raisons étrangères à ces principes.

Par contraste, selon l'approche « à partir de l'intérieur » de Darwall, la théorie de l'action juste est liée directement à notre conception du caractère intègre. C'est même à partir de celle-ci que celle-là est développée. Dans cette optique, une fois établi quels sont les principes qui guident l'action d'une bonne personne - une personne de caractère intègre — nous avons déjà, et immédiatement, notre théorie de l'action juste. Car les principes qui déterminent ce qui est bien et ce qui est mal sont précisément, et uniquement, les principes qu'utilise dans ses délibérations la personne au caractère intègre. Selon l'approche darwallienne, il n'y a pas de différence possible entre ce qui guide les agents de caractère intègre et ce qui est vraiment bien ou mal. Il n'y a donc pas d'écart entre les deux théories du caractère et de l'action, comme on en trouve chez le conséquentialiste. En somme, parce que les agents au caractère intègre adhèrent à des restrictions déontologiques (rappelons que chaque parti admet cela, selon Darwall), ces restrictions font partie de la théorie correcte de l'action juste. Nous avons donc établi les restrictions déontologiques comme élément important de la morale en passant par une prémisse acceptée même par les conséquentialistes.

Selon Darwall, nous devons comprendre la morale de cette façon c'est-à-dire "à partir de l'intérieur » - si nous espérons trouver une place pour les restrictions déontologiques. Mais même s'il est possible de dériver les normes déontologiques d'une telle approche de l'éthique, nous devons

13. Ibid., p. 308. 
espérer, je crois, que ce n'est pas la seule manière de les fonder. Car Darwall ne nous offre pas, lui non plus, une explication intuitivement satisfaisante du phénomène en question. Même si la théorie de Darwall implique vraiment qu'il y ait des contraintes déontologiques au sein de la morale, elle confère un statut dérivé à ces contraintes, comme, du reste, à tous les principes de conduite, à l'exception d'un seul. Selon Darwall, notre souci primordial en tant qu'agent moral est celui de préserver notre intégrité morale : c'est là l'exigence morale fondamentale. Toute autre exigence morale doit donc découler de ce réquisit de base. Les types de conduite qui sont interdits le sont parce $q u$ 'agir ainsi constitue une violation de ce devoir fondamental. Mais tout comme la conception de Nagel, cette approche ne semble pas situer adéquatement le mal qui caractérise certaines formes de conduite ou de traitement d'autrui. La raison pour laquelle il est mal de torturer un enfant doit, semble$\mathrm{t}$-il, se trouver dans le tort qui lui est fait, et non dans le préjudice que le tortionnaire cause à sa propre intégrité morale. Cette seconde identification de la source du mal ne rend pas justice à notre compréhension antécédente de la base de la contrainte, qui, croyons-nous vaguement mais avec confiance, doit plutôt provenir de considérations du premier type.

On pourrait même affirmer, de façon plus générale, que nos devoirs moraux fondamentaux doivent être envers les autres, et non envers soi-même. Sans nier qu'il y ait aussi des devoirs du second type, la morale n'existerait tout simplement pas s'il n'y avait pas d'autres personnes avec des besoins, des intérêts et des droits qu'il faut concilier avec ceux qu'on possède soi-même. C'est ce fait primordial qui est à l'origine de la morale. Les restrictions déontologiques nous paraissent comme une expression significative de cette idée, une illustration de l'importance des intérêts des autres pour la conduite morale. Darwall, cependant, essaie de justifier les contraintes déontologiques en les plaçant « à l'intérieur » de l'agent, au lieu de les baser sur des considérations qui ont à voir avec les victimes potentielles. Spécifiquement, il les défend en les reliant à ce devoir, supposément de première importance, de maintenir sa propre intégrité morale. Les normes déontologiques n'ont donc pas de fondements plus sûrs que ce principe de base, qui parait cependant mal représenter les préoccupations centrales de la morale. Pour ces raisons, même si Darwall réussit à soutenir les normes déontologiques par cette ligne de pensée, cette explication ne nous satisfera probablement pas. Elle ne nous aide pas à comprendre notre conviction qu'il est mal de traiter les autres de certaines manières.

Nagel ${ }^{14}$ et Darwall nous proposent de chercher dans le raisonnement pratique de l'agent les raisons pour lesquelles sa conduite est condamnable. Mais notre investigation suggère que c'est en concentrant notre attention sur la victime que nous avons le plus de chances de comprendre la base des restrictions déontologiques. De fait, une telle piste de réflexion paraît mieux répondre aux intuitions déontologiques qui motivent notre étude. Il demeure néanmoins

14. Nagel, 1986. 
qu'une approche centrée sur la victime pourrait avoir du mal à se distinguer de l'optique conséquentialiste, car cette dernière semble proposer la façon la plus directe et la plus simple de tenir compte des intérêts des victimes. Y a-t-il toutefois une autre façon de tenir compte de la victime, qu'on peut distinguer clairement de l'approche "agrégative » du conséquentialisme ? Je propose de diriger notre attention vers la conception que nous nous faisons du statut moral protégé dont jouit chaque personne. Cette idée ne se prête guère à une interprétation agrégative, et son investigation me paraît susceptible de faire progresser notre réflexion. S'il était possible d'élucider davantage la nature de ce statut et les raisons pour lesquelles nous l'attribuons aux personnes, je crois que nous comprendrions mieux la source des normes déontologiques ${ }^{15}$.

Une mine d'idées qu'il est naturel d'exploiter dans cette enquête est, bien sûr, l'œuvre d'Emmanuel Kant. Sans traiter des idées kantiennes avec trop de détails, essayons de voir en termes généraux l'aide que celles-ci pourraient nous apporter. Commençons donc par affirmer que l'humanité ou la nature rationnelle a un statut spécial, même unique, en tant que fin en soi. Elle n'a pas de prix, mais possède plutôt une dignité située au-delà de tout prix. Poursuivre toute fin subjective aux dépens de la nature rationnelle ellemême constitue donc non simplement une évaluation erronée mais une erreur plus sérieuse, une confusion profonde. La nature rationnelle est la condition de toute valeur : toute fin subjective a de la valeur uniquement parce qu'elle a été choisie par un agent rationnel. La nature rationnelle ne doit donc jamais être sacrifiée au profit d'un but subjectif et contingent : agir ainsi revient à poursuivre une fin au prix de détruire ce qui a conféré la valeur à cette fin en premier lieu. Il y a donc une priorité lexicale de la nature rationnelle sur toute fin qu'elle peut sélectionner.

Ces idées expliqueraient une partie des données déontologiques que nous cherchons à mieux comprendre. Par exemple, elles expliqueraient pourquoi il est mal de tuer quelqu'un pour son propre plaisir ou celui des autres, peu importe l'intensité du plaisir que ce genre d'acte puisse susciter. Le plaisir est un but purement subjectif, il ne pourrait donc jamais justifier la destruction de la nature rationnelle en la personne de la victime. Le plaisir n'est ici qu'un exemple : le même argument interdirait qu'on tue pour avancer n'importe quel but subjectif, qu'il s'agisse du sien ou de celui d'autres personnes. Nous pourrions de cette manière rendre compte de certains types de prohibitions déontologiques : celles où le meurtre est une tentative de promouvoir des fins purement subjectives.

Mais il y a d'autres cas qui sont moins bien traités par l'idée de la priorité de l'humanité sur toute fin qu'elle puisse choisir. Car il est possible d'interpréter l'approche kantienne comme rien d'autre qu'une axiologie, c'est-à-dire comme rien d'autre qu'un moyen distinctif d'évaluer la valeur

15. J'ai modifié ce paragraphe suite aux commentaires d'un évaluateur anonyme, que je voudrais encore une fois remercier. 
des états de choses. David Cummiskey ${ }^{16}$ développe cette interprétation, avec la conséquence inattendue que les idées kantiennes s'intègrent facilement à une théorie conséquentialiste, formant même la base d'une version nouvelle de cette théorie morale. Dans l'interprétation axiologique de l'approche kantienne, les remarques déjà résumées indiquent seulement que la nature rationnelle a beaucoup plus - même incomparablement plus - de valeur intrinsèque que toute autre chose. Ce serait donc une grave erreur que de préférer un état de choses par lequel une fin autre que la nature rationnelle est priorisée aux dépens de celle-ci : un tel état de choses ne pourrait avoir autant de valeur que celui par lequel la nature rationnelle est préservée. Cependant, rien dans cette axiologie n'exclut la possibilité qu'elle serve de base à une conception téléologique ou conséquentialiste de la morale. Comme nous l'avons constaté, aucune axiologie de la valeur intrinsèque et impersonnelle n'est incompatible avec une telle approche de la théorie morale : le conséquentialisme peut adopter n'importe quelle axiologie de ce type. Il y a donc une théorie conséquentialiste basée sur cette axiologie "kantienne ». Elle nous ordonne, comme chaque forme de conséquentialisme, de toujours maximiser la valeur intrinsèque, ajoutant que les jugements de valeur comparatifs doivent toujours obéir à la priorité lexicale de l'humanité sur toute autre fin.

Cette théorie morale n'est pas déontologique. En effet, elle nous oblige à minimiser la destruction de la nature rationnelle quand c'est en notre pouvoir de le faire, même si cela implique que nous détruisions nous-mêmes la nature rationnelle. (Il en va de même pour tout préjudice causé à la nature rationnelle : selon la théorie conséquentialiste, nous sommes obligés de causer de tels préjudices si c'est là le seul moyen de prévenir d'autres atteintes plus graves à l'humanité.) Assurément, le fait de détruire ou de causer des préjudices à plusieurs êtres rationnels est pire, selon cette axiologie, que le fait de détruire ou de causer préjudice à une seule personne : il est donc obligatoire de générer, quand on a le choix, des conséquences du second type de préférence à celles du premier type. Par exemple, selon cette théorie " conséquentialiste kantienne » et en considérant l'hypothèse que la bombe des terroristes détruira plusieurs agents rationnels, il serait obligatoire de détruire la nature rationnelle en la personne de l'enfant afin de prévenir ces autres morts plus nombreuses.

Ce résultat nous démontre que les idées kantiennes, du moins si nous les laissons au niveau des slogans, ne suffisent pas à mener à une approche distinctement déontologique de la morale. Il est toujours possible de les interpréter comme de simples affirmations axiologiques pouvant être intégrées dans un cadre moral conséquentialiste. Vues de cette manière, cependant, elles donnent lieu à des conclusions morales que le déontologiste trouvera répugnantes. Il est faux, donc, que tout acte qui viole une restriction

16. Dans Cummiskey, 1990 et 1996. 
déontologique est incompatible avec toutes les interprétations plausibles de l'idée selon laquelle l'humanité prime sur toute autre fin. Cela veut dire que nous n'avons pas encore trouvé une explication complète de nos convictions déontologiques : faire appel à ces notions kantiennes ne suffit évidemment pas. Bien sûr, Kant lui-même n'approuverait probablement pas cette interprétation de ses propos ${ }^{17}$. Mais il faudra quand même préciser davantage l'interprétation que nous avons en tête si nous voulons utiliser ces idées kantiennes pour expliquer ou fonder des normes déontologiques.

Considérons maintenant la perspective décrite ces dernières années par Frances $\mathrm{Kamm}^{18}$ et Thomas Nagel ${ }^{19}$, qui s'avérera prometteuse et que nous résumerons maintenan $\mathrm{t}^{20}$. Selon eux, chaque système moral exprime une conception particulière du statut moral des personnes. Un cadre moral qui interdit de traiter les gens de certaines façons, même quand les traiter ainsi servirait le bien-être collectif, exprime une conception des personnes comme inviolables. Il faut souligner que l'inviolabilité est une fonction de ce qu'il est permis de nous faire, et non de ce qui nous est fait effectivement. Comme elle réside dans la morale elle-même, l'inviolabilité ne peut être créée ni influencée par ce que nous faisons : il nous est impossible de maximiser l'inviolabilité au moyen de nos actes. Kamm et Nagel présentent des arguments à l'effet que notre possession d'un tel statut moral est pour nous un bénéfice : Kamm suggère même qu'elle rend le monde meilleur, parce que l'univers contient alors des entités plus sublimes ${ }^{21}$.

De toute façon, selon Kamm et Nagel, l'inviolabilité est une véritable valeur objective. S'il était acceptable de maltraiter une personne pour épargner le même sort à plusieurs autres personnes, cela voudrait dire que personne n'est inviolable en fin de compte. Et cela serait une mauvaise chose pour nous, car notre inviolabilité est intrinsèquement valable. Nous avons donc une explication de la raison pour laquelle, par exemple, il est mal de torturer même dans le but de promouvoir le bien-être total collectif. Affirmer le contraire serait inacceptable, car cela impliquerait que nous ne sommes plus réellement inviolables. En faisant appel à la valeur de l'inviolabilité, Kamm et Nagel suggèrent que les normes déontologiques expriment une forme de valeur objective et neutre-quant-à-l'agent, qui ne se prête pas à une analyse conséquentialiste et qui n'existerait même pas sous une morale con-

17. Cummiskey en convient, mais il soutient que l'interprétation «conséquentialiste kantienne » représente quand même une importante contribution à la théorie morale. De plus,

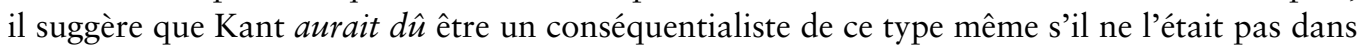
ses convictions morales plus spécifiques.

18. Kamm, 1996, surtout le chapitre 10.

19. Nagel, 1994 et 1995 , $\mathbb{S}$ I-III.

20. Nagel est conscient que l'explication qu'il offre désormais est orientée dans une toute autre direction que celle qu'il proposait auparavant. Nagel, 1995, p. 89, note 2.

21. Kamm, 1996, p. 272. 
séquentialiste. On a donc ici une avenue potentiellement puissante pour l'explication et, ultimement, la défense du déontologisme.

Soulignons quelques points forts de cet appel à la valeur de l'inviolabilité qu'expriment les systèmes moraux déontologiques. Primo, Kamm et Nagel semblent avoir raison de faire valoir que notre statut moral nous importe, quoi qu'il nous arrive en fait. Comme l'exprime Nagel, « il serait horrible d'être torturé ; mais être torturé et être également quelqu'un qu'il n'est pas mal de torturer serait pire encore $»^{22}$. Nagel a correctement identifié une considération distincte qui a son propre poids, qui s'ajoute à l'importance de ce qui nous est fait. Car nous valorisons notre statut moral même quand il est violé. Nous valorisons notre statut d'êtres ayant des droits, indépendamment du fait que nous pourrions ne jamais les faire valoir, ou ne pas en ressentir le besoin. Parce que cette considération a sa propre importance, indépendamment de tout autre élément pertinent au choix moral, Kamm et Nagel ont raison d'attirer notre attention sur cet aspect du choix auquel nous faisons face dans un cas comme celui des terroristes. Nous devrions tenir compte, non seulement de ce qui arrivera à chaque personne impliquée dans le scénario, mais aussi du statut moral que notre décision attribuera implicitement à chacun de nous. Si nous décidons qu'il est permis, et même obligatoire, de torturer l'enfant pour prévenir l'attentat, nous devrions réfléchir aux implications de ce jugement pour l'inviolabilité de chaque personne.

Un aspect important de la proposition de Kamm et Nagel, c'est qu'ils attribuent une valeur intrinsèque - et non simplement instrumentale — à la possession des droits et à l'inviolabilité qu'ils nous accordent. Ceci rend possible l'explication du fait que nous tenons toujours à nos droits même quand ils ne sont pas respectés, et donne donc un avantage considérable à leur théorie en comparaison avec des justifications instrumentales des droits. Voyons brièvement, en examinant un exemple sophistiqué de ces dernières, pourquoi les théories de ce type ont du mal à n'attribuer aucune valeur aux droits si ces derniers ne sont pas respectés. Dans son article de $1978^{23}$, T. M. Scanlon propose un modèle « à deux niveaux » de la morale, qui tente de justifier certains droits (et donc, dans le langage de Kamm et de Nagel, une certaine forme d'inviolabilité). Mais dans sa conception (indépendamment, peut-être, de ce que l'auteur entend faire valoir), la valeur de la possession des droits est comprise uniquement comme la valeur instrumentale du respect de ces droits. Pour que la valeur de la possession des droits se réalise, il faut la coopération générale des autres : sans cela, les droits ne servent à rien. La théorie de Scanlon diffère donc, dans un aspect crucial, de celle de Kamm et de Nagel.

22. Nagel, 1995, p. 93.

23. Scanlon propose une toute autre théorie dans son livre récent (1999). Néanmoins, l'argument que l'on trouve dans l'article cité dans le texte demeure intéressant, même si son auteur n'y adhère plus et que cet article ne représente qu'une étape de sa pensée. 
Scanlon motive sa théorie des fondements et du fonctionnement des droits en argumentant qu'il doit y avoir une valeur ou un but que les droits promeuvent. "Les droits eux-mêmes ont besoin qu'on les justifie d'une certaine manière, et comment le faire autrement qu'en faisant appel aux intérêts humains que leur reconnaissance promeut et protège ? "24. Dans la recherche de la fondation des droits, nous devrions donc chercher des intérêts que les droits peuvent protéger ou promouvoir. Scanlon veut cependant éviter une approche relevant de "l'utilitarisme de la règle ", car elle exige, selon lui, une conformité quasi totale aux règles pour que les valeurs visées soit réalisées ${ }^{25}$. Il propose de fonder des droits à l'aide d'un argument en trois étapes, qui comporte une partie empirique. D'abord, nous examinons comment les individus et les institutions se comporteraient en l'absence d'un certain droit. Puis, nous évaluons si cet état de choses serait acceptable du point de vue des intérêts humains substantiels. Enfin, dans le cas où nous jugeons cet état de choses inacceptable, nous montrons comment la situation serait différente si le droit en question était bel et bien accordé. Ces trois étapes constituent l'argument en faveur de l'existence d'un droit particulier. Constatons que « cette perspective des droits est conséquentialiste dans un sens large, en ce qu'elle croit que les droits sont justifiés par un appel aux états de choses qu'ils promeuvent ${ }^{26}$.

Il faut comprendre quel genre d'états de choses les droits promeuvent. N'y a-t-il qu'un seul type de valeur ou de but que les droits, de par leur nature même, assurent ? Ou existe-t-il pour chaque droit une conséquence valorisée qui lui est spécifique ? La réponse de Scanlon est que les droits sont unifiés : il y a une valeur générale que tous les droits promeuvent, peu importe le contenu spécifique de chaque droit. Cette valeur se classe dans une catégorie des intérêts humains que les utilitaristes classiques sous-estiment, selon Scanlon. C'est-à-dire qu'une conception des intérêts humains « doit inclure, non seulement les choses qui peuvent arriver aux individus mais aussi les facteurs qui affectent la capacité des individus de déterminer ce qui arrivera ${ }^{27}$. Cette idée ressemble à celle de Kamm et de Nagel dans une certaine mesure : Scanlon maintient lui aussi que ce qui arrive aux gens est seulement une partie de ce qui compte pour eux. Mais sa conception de ce qui est omis par une approche qui ne considère que ce qui a lieu est différente, semble-t-il, de celle de Kamm et de Nagel. Pour Scanlon, les droits protègent précisément ces intérêts que nous avons à déterminer ce qui nous arrive ou ce que nous faisons. Les droits, quels que soient les domaines particuliers d'activité qu'ils gouvernent, nous donnent une mesure de contrôle sur notre vie, que nous valorisons. Donc, résume Scanlon, « l'argument pour les droits dérive en

24. Scanlon, 1978, p. 74.

25. Ibid., p. 75.

26. Ibid., p. 85.

27. Ibid., p. 76, mes italiques. 
grande partie du but qui consiste à promouvoir une distribution acceptable du contrôle sur des facteurs importants dans nos vies ${ }^{28}$.

Ces conséquences heureuses, cependant, seront réalisées uniquement si ces droits sont en fait honorés. Si les autres ne respectent pas mes droits tels que générés par la théorie de Scanlon, alors, dans les faits, je n'ai pas le contrôle sur d'importants aspects de ma vie, contrôle que l'approche de Scanlon tente de nous garantir. Je n'ai aucun contrôle sur ce qui m'arrive ou sur ce que je fais si les autres entravent mon action, par exemple en me blessant ou en me tuant. Il paraît donc que Scanlon nous offre un argument en faveur de l'observance des droits, mais qui n'appuie pas l'existence de ces droits considérée indépendamment de leur observance. L'argument en faveur des droits repose sur les valeurs que ces droits apportent. Si les droits n'amènent pas ces bonnes conséquences, alors l'argument offert par Scanlon ne s'applique plus et nous n'avons plus de raison de valoriser les droits. Mais la valeur en question - le contrôle - dépend effectivement de ce que les droits promulgués soient réellement respectés. S'ils ne le sont pas, nous ne pouvons plus identifier quoi que ce soit comme étant la valeur promue par les droits, et la simple possession de ces droits n'est donc plus valable. En évitant d'accorder une valeur intrinsèque à la possession des droits, Scanlon se met en effet dans la même situation que les théories relevant du " conséquentialisme de la règle " qu'il a critiquées au début de son article. Par contraste, Kamm et Nagel maintiennent que la possession des droits ou l'inviolabilité ont une valeur indépendante, qui persiste même dans des conditions de non-conformité générale ou de non-respect par les autres. Ceci représente un élément neuf et, je crois, un progrès dans la théorie du déontologisme.

Il est plus difficile d'expliquer le deuxième aspect important du modèle de l'inviolabilité. Cette conception trouve dans la valeur intrinsèque et neutrequant-à-l'agent une base pour les normes déontologiques. Mais la valeur à laquelle elle fait appel n'est néanmoins pas sujette à ce qu'on pourrait appeler " le maître argument » conséquentialiste contre les restrictions déontologiques. Cet argument fonctionne comme suit. Imaginons un cas où tuer est interdit par une prohibition déontologique : par exemple, un cas où il faudrait tuer pour prévenir d'autres meurtres plus nombreux. Supposons que nous tentions d'expliquer pourquoi il est quand même mal de tuer dans ce cas en faisant appel à une valeur positive ou négative, telle que la valeur positive de la vie ou la valeur négative de la mort. Peu importe la valeur spécifique que nous citons, une fois que nous donnons une explication de cette forme, nous sommes aux prises avec le maître argument conséquentialiste. Car le conséquentialiste répliquera que l'explication que nous venons de donner invalide le jugement qu'elle est censée expliquer. Si nous tenons tant à préserver la vie ou à minimiser les morts - comme le suggère notre tentative d'explication nous devrions être préparés à tuer quand cela aura pour effet de minimiser les

28. Ibid., p. 86 . 
morts ou de préserver autant de vies possibles. Quelquefois, on promouvra mieux la valeur de la préservation de la vie en acceptant de tuer plutôt qu'en s'y refusant, si cette dernière attitude permet par exemple à d'autres meurtriers d'accomplir leurs projets.

Le conséquentialiste affirmera donc que nos propres priorités, telles que révélées par notre discours sur ce qu'il y a de mal dans le fait de tuer, nous amènent au conséquentialisme plutôt qu'aux normes déontologiques. Autrement dit, l'explication que nous avons offerte ne soutient pas une restriction déontologique contre le meurtre. Nous avons déjà vu la possibilité d'une réplique comme celle-ci en discutant l'interprétation axiologique des idées kantiennes. Une fois dans le domaine de l'axiologie - des règles pour l'évaluation des états de choses - nous devons admettre qu'il faille parfois agir, dans une certaine mesure, à l'encontre d'une valeur afin d'en maximiser la quantité totale. Cela est vrai pour la valeur de la nature rationnelle comme pour toute autre valeur que nous pourrions citer, semble-t-il.

L'inviolabilité, cependant, bien qu'elle soit une valeur, n'en est pas une que nous pouvons maximiser par nos actes. L'inviolabilité réside dans la conception même du système moral, et n'est donc pas susceptible d'être promue ni empêchée par ce que nous faisons. Le conséquentialiste ne peut pas donc nous conseiller de violer l'inviolabilité afin de rapporter encore plus d'inviolabilité. Car nos actes n'ont pas d'effet sur la « quantité » d'inviolabilité présente au monde (si tant est que parler en termes de quantité a un sens dans ce contexte). Ou bien nous sommes inviolables - si la morale nous offre effectivement le genre de protection qu'expriment les restrictions déontologiques - ou bien nous ne le sommes pas - si, au contraire, le conséquentialisme représente la vérité sur le plan moral. Ce que nous faisons ne change rien à cet égard. Il est donc impossible pour le conséquentialiste d'utiliser son maître argument contre la suggestion que les normes déontologiques expriment un statut d'inviolabilité qui est d'une grande valeur. Un appel à cette valeur ne peut être transformé en piège contre le déontologiste, contrairement à toute autre valeur ordinaire (c'est-à-dire, toute valeur susceptible d'être maximisée ou minimisée par nos actes).

Ce grand avantage que possède l'approche basée sur l'inviolabilité le fait de ne pas être sujette au maître argument conséquentialiste — engendre, cependant, une difficulté qui semble également particulière à cette perspective. Précisément parce que l'inviolabilité n'est pas affectée par nos actes, le raisonnement suivant pourrait paraître irrésistible. "Je dois décider si je vais torturer cet enfant ou laisser plusieurs personnes mourir dans l'attentat. Je n'ai pas d'autre choix que ces deux possibilités. Je n'aimerais pas affirmer qu'il m'est permis de torturer l'enfant dans cette situation, car ce serait dire que nous ne sommes pas inviolables, et dans ce cas nous aurions perdu quelque chose d'une grande valeur. Mais quoi qu'il en soit, il est un fait (que nous sommes inviolables ou que nous ne le sommes pas) qui ne changera pas, quoi que je fasse. Laquelle de ces deux options est correcte, est une fonction du 
système moral, et, en regard de ma propre décision, ce facteur est une constante inconnue dans l'équation. Je devrais plutôt me concentrer, donc, sur les variables que je suis en mesure d'affecter : les valeurs ordinaires telles que la valeur positive de la vie et la valeur négative de la mort. Que nous soyons inviolables ou non, je peux en tout cas conclure avec confiance que ce sera pour le mieux si je préviens ces morts en torturant l'enfant. En agissant ainsi je maximiserai les valeurs ordinaires comme celle de la vie, sans nuire à notre statut d'inviolabilité, qui demeurera intact quoi que je fasse ».

Ce raisonnement, qui rappelle l'approche du problème de Newcomb qui favorise le choix des deux boîtes, concède un point sur lequel Kamm et Nagel insistent : nous perdrions quelque chose de très important si nous étions prêts à affirmer qu'il est permis de tuer quand les conséquences de cet acte sont suffisamment bonnes. Car cela voudrait dire que nous ne sommes plus inviolables : nous aurions perdu ce statut et la dignité qu'il nous confère. En contraste avec le conséquentialiste, donc, ce raisonnement ne conteste pas le jugement qu'il n'est pas permis de tuer dans cette situation. Il traite la question de l'inviolabilité plutôt comme une " boîte noire » dont le contenu est en tout cas déjà fixe : quoi que l'agent fasse, cette «boîte " contiendra soit l'inviolabilité, soit un statut différent. L'agent tourne donc son attention vers la seconde "boîte ", c'est-à-dire vers les valeurs ordinaires qu'il peut maximiser s'il le décide. Il choisit de les maximiser, même si cela exige qu'il tue ou qu'il torture, en pensant, " cela sera pour le mieux ». Et dans un certain sens il a raison. Par hypothèse, il maximisera les valeurs à sa portée : il amènera le meilleur état de choses possible, vu d'une perspective impartiale. Cette solution peut donc paraître n'imposer aucun coût : choisir de maximiser la valeur autant que possible sans tirer la conclusion que la morale exige cette ligne de conduite. Tirer cette conclusion équivaudrait à renoncer à nos prétentions à l'inviolabilité, et nous préférerions éviter cela ; mais en attendant, maximisons le bien-être collectif.

Il vaut la peine de souligner de façon explicite deux aspects importants de ce raisonnement. D'abord, il importe de remarquer que ce raisonnement ne poursuit pas le but traditionnel de la théorie morale, c'est-à-dire celui d'établir une conception particulière de ce qui est moralement permis, quitte à en altérer notre conception antérieure. C'est plus ou moins l'ambition de tout théoricien de l'éthique, et certainement celle du conséquentialiste. Ce dernier veut nous convaincre de sa propre conception de ce qui est permis, une conception incompatible avec celle des partisans de l'inviolabilité. Le conséquentialiste conteste les affirmations des déontologistes et préconise une conception rivale du système moral. Par contraste, le raisonnement que nous venons de décrire ne conteste pas l'idée que nous sommes inviolables. Celui qui est tenté par ce raisonnement concédera volontiers que nous sommes inviolables, c'est-à-dire, qu'il est moralement interdit d'agir comme il propose d'agir. Contrairement au conséquentialisme, donc, ce raisonnement ne s'oppose pas au déontologisme en niant ce qu'affirme ce dernier. Son 
" opposition » est d'un autre genre, ce qui nous amène au deuxième aspect de ce raisonnement qu'il faut souligner.

Au lieu de contester la conception déontologiste de ce qui est permis, ce raisonnement propose plutôt de ne tenir aucun compte, dans le choix d'une ligne de conduite, de ce qui est moralement permis. Notre agent n'est pas guidé par de telles considérations, même partiellement, mais plutôt et uniquement par le désir de faire ce qui sera pour le mieux, c'est-à-dire en ayant pour but de maximiser la valeur. Ainsi, l'agent ne respecte pas par son action la conclusion morale qu'il est prêt à affirmer par ailleurs. L'agent n'assigne pas pour autant à cette conclusion morale un certain poids qui s'avérerait en fin de compte inférieur au poids d'autres considérations. Plutôt, il en fait complètement abstraction en décidant de ce qu'il va faire. C'est dans ce sens qu'il rejette les recommandations des déontologistes et de ceux qui mettent l'emphase sur notre inviolabilité. Même s'il accepte leurs affirmations, il ne leur accorde aucune importance en tant que guide de l'action.

L'approche de Kamm et de Nagel peut sembler inviter un tel traitement, et ce pour deux raisons. Le fait que l'inviolabilité elle-même ne soit pas affectée par ce que nous faisons leur permet d'éviter le maître argument conséquentialiste. Mais ce fait rend également possible l'attitude de notre agent hypothétique, qui pense ainsi : " notre statut d'inviolabilité demeurera intact quoi que je fasse ». En second lieu, Kamm et Nagel insistent sur la valeur que conserve l'inviolabilité indépendamment de son expression dans l'action. Ils font ainsi valoir un aspect du déontologisme qui ne se résume pas à son rôle de guide de l'action. En plus de ce rôle, un système moral déontologique a aussi une valeur qui est indépendante de ce dernier. Nous sommes accoutumés à ne considérer ou évaluer les théories morales qu'en tant que guides de l'action, mais cette perspective tente d'attirer notre attention sur une autre dimension du déontologisme, une autre contribution qu'il est susceptible d'apporter. C'est même sur cette dimension qu'elle insiste. Le hic, c'est qu'une telle ligne d'argument peut sembler amenuiser la pertinence du jugement moral à l'égard de la décision pratique. Si la morale n'est plus uniquement un guide de la conduite, elle risque de sembler s'effacer d'elle-même ${ }^{29}$.

Cependant, nous devrions rejeter le raisonnement de notre agent hypothétique, que les considérations que nous venons d'évoquer ne sauraient justifier. Rappelons que cet agent affirme croire en notre inviolabilité, mais qu'il n'accorde, au moment de faire des choix au cours de l'action, aucun rôle effectif à cette supposée croyance. Il prend sa décision selon un tout autre principe, tout en sachant (par hypothèse) que cette décision va à l'encontre de la morale. Il propose donc sciemment d'agir de façon immorale : d'agir contrairement à ce que la morale exige selon son propre jugement, de faire quelque chose que la morale interdit fermement. Dans ses choix et son

29. Il est intéressant de comparer cette ligne de pensée avec l'argument de Bernard Williams selon lequel le conséquentialisme s'efface de lui-même (Williams 1973, p. 134-135). 
action, il ne reconnaît aucun poids à ce que commande la morale. En bref, cet agent propose de traiter son verdict moral concernant les options qui s'offrent à lui comme étant hors sujet et non pertinent eu égard à son choix. Mais une telle attitude n'est nullement une conséquence de la ligne de pensée qui insiste sur l'inviolabilité accordée par un système déontologique. Selon cette ligne d'argument, la morale n'est pas uniquement un guide de la conduite : elle possède une valeur indépendante de son influence sur la conduite. Mais cela ne veut pas dire que la morale n'est plus un guide de l'action! Attirer notre attention sur une dimension supplémentaire de la morale, ce n'est pas nier ses fonctions traditionnelles. Sous l'optique de l'inviolabilité, la morale continue de prescrire certaines actions et d'en interdire d'autres, et de réclamer toute l'importance qui lui est normalement attribuée eu égard au choix pratique ${ }^{30}$. Notre agent a mal compris cette perspective s'il croit qu'elle a renoncé à toute aspiration à guider le choix.

En fait il paraît y avoir au moins deux interprétations possibles de ce que pense notre agent : ou bien il croit vraiment que nous sommes inviolables, ou bien il ne le croit pas véritablement. Suivant la seconde possibilité, il ne serait déontologiste qu'en paroles. Sa vraie morale serait plutôt le conséquentialisme : c'est ce que son action révèle. Il ne veut pas l'affirmer, car il comprend que ce serait nous priver du statut de l'inviolabilité. Mais ses choix démontrent néanmoins son adhésion à une morale conséquentialiste. Dans ce cas, notre agent est hypocrite. S'il n'est pas d'accord avec les affirmations du déontologiste, il devrait admettre ce fait et entreprendre avec ce dernier une discussion sur la théorie morale. (Nous avons déjà souligné que le raisonnement de notre agent n'est pas formulé en ces termes.)

Il y a aussi la première possibilité : que notre agent croit vraiment à notre inviolabilité. C'est-à-dire qu'il juge sincèrement qu'il est interdit d'agir comme il propose de le faire. Néanmoins, il considère ce fait comme hors sujet en ce qui concerne son propre choix : il effectuera ce dernier sur la base d'un tout autre principe. Dans ce cas, tous les théoristes moraux qui croient à l'importance des verdicts moraux dans le raisonnement pratique devraient unanimement s'élever contre cette attitude. Selon la plupart des théoristes moraux, quelles que soient les théories particulières qu'ils défendent, la morale est une considération de prime importance quand il s'agit de faire un choix, sinon la considération la plus importante de toutes. Si l'on est tenté de défendre notre agent en citant le fait qu'il agit pour le bien, rappelons que, pour cet agent, la question morale est distincte de celle du bien, quand ce dernier se voit accordé un sens non spécifiquement moral. Cet agent considère la question morale comme déjà résolue en faveur de l'interdiction de la torture de l'enfant, mais il propose de l'entreprendre quand même, et ce, sans

30. Pour une discussion et une défense de l'importance de la morale à cet égard, voir Stroud, 1998. 
accorder la moindre importance au fait que la morale l'interdit. Cela devrait inquiéter, pour le moins, tout théoriste moral ${ }^{31}$.

En terminant, rappelons que nous avons relevé plusieurs aspects fort prometteurs de ce genre d'explication qui fait appel à la valeur de l'inviolabilité que seule une morale déontologique nous accorde. Elle affirme la valeur intrinsèque de notre statut moral. Elle est à l'abri du maître argument conséquentialiste. Et, contrairement aux tentatives de Nagel (dans son livre de 1986) et de Darwall, elle semble situer la source des normes déontologiques au bon endroit : plutôt dans notre rôle de patient - de victime potentielle - que dans celui d'agent. Elle s'avère donc bel et bien digne de plus amples développements et raffinements. Car il faudrait, bien sûr, préciser davantage plusieurs choses à propos de l'inviolabilité pour en arriver à une explication complète des contraintes auxquelles nous croyons. Primo, qu'est-ce qui détermine la portée de l'inviolabilité, c'est à dire qui bénéficie de ce statut ? En second lieu, comment devons-nous choisir entre différentes conceptions de l'inviolabilité ? Si différents systèmes moraux expriment des conceptions divergentes de notre statut moral, comment faire un choix parmi eux ? Il ne faut pas supposer que nous préférerons le système moral qui accorde le plus d'inviolabilité : cela pourrait rendre difficile de trouver des entités qu'il est permis de manger ! Finalement, quel poids devrions-nous accorder à l'inviolabilité en comparaison avec d'autres valeurs avec lesquelles elle peut entrer en conflit ? Il y a donc du travail à faire dans ce domaine. Mais nous serions en droit, au moins, de nous croire sur la bonne voie en développant une explication plus complète formulée en ces termes. Les premiers pas de notre investigation n'auront pas été vains ${ }^{32}$.

\section{Bibliographie}

Cummiskey, David, "Kantian Consequentialism », Ethics, 100, 1990, p. 586-615.

Cummiskey, David, Kantian Consequentialism, Oxford, Oxford University Press, 1996.

Darwall, Stephen L., "Agent-Centered Restrictions from the Inside Out ", Philosophical Studies, 50, 1986, p. 291-319.

Fried, Charles, Right and Wrong, Cambridge (Mass.), Harvard University Press, 1978.

Kamm, F. M., Morality, Mortality. Volume II : Rights, Duties, and Status, New York, Oxford University Press, 1996.

31. J'aimerais remercier l'évaluateur anonyme qui m'a encouragée à étoffer cette dernière partie du texte.

32. Cet article tire son origine d'une conférence que j'ai donnée à l'Université Princeton. J'aimerais remercier ceux qui étaient présents lors de cette occasion pour leurs commentaires. Je voudrais également remercier Pierre Charette pour les corrections qu'il a apportées au texte français. 
Nagel, Thomas, The View From Nowhere, New York, Oxford University Press, 1986.

Nagel, Thomas, "La valeur de l'inviolabilité », Revue de métaphysique et de morale, 99, 1994, p.149-166.

Nagel, Thomas, "Personal Rights and Public Space ", Philosophy \& Public Affairs, 24, 1995, p. 83-107.

Scanlon, T. M., "Rights, Goals, and Fairness ", dans Scheffler, S., dir., Consequentialism and its Critics, Oxford, Oxford University Press, 1988, publié la première fois dans Hampshire, S., dir., Public and Private Morality, Cambridge, Cambridge University Press, 1978.

Scanlon, T. M., What We Owe to Each Other, Cambridge (Mass.), Harvard University Press, 1999.

Scheffler, Samuel, The Rejection of Consequentialism, Oxford, Clarendon Press, 1982.

Scheffler, Samuel, «Introduction », dans Scheffler, S., dir., Consequentialism and its Critics, Oxford, Oxford University Press, 1988.

Stroud, Sarah, "Moral Overridingness and Moral Theory", Pacific Philosophical Quarterly, 79, 1998, p. 170-189.

Williams, Bernard, "A Critique of Utilitarianism», dans Smart, J. et Williams, B., Utilitarianism : For and Against, Cambridge, Cambridge University Press, 1973. 